\title{
Deep Learning techniques for effective diagnosis of Alzheimer's disease using MRI images
}

\author{
Prajakta Tambe ${ }^{1}$, Rutuja Saigaonkar ${ }^{l}$, Nidhi Devadigal and Ms. Pallavi H. Chitte ${ }^{l}$ \\ ${ }^{1}$ Department of Computer Engineering, University of Mumbai, Ramrao Adik Institute of Technology, India.
}

\begin{abstract}
The determination of Alzheimer's disease (AD) from neuroimaging data such as MRI has been immensely researched over the last few years. Recent advances in deep learning from a computer perspective have advanced in that research. However, the general limitations of such algorithms depend on the large number of training images, as well as the need to carefully perform the construction of deep networks. In past few days deep learning strategies have found great achievement in the analysis of medical imaging. But very little has been done in the use of deep learning strategies to turn up and differentiate Alzheimer's disease. We are building a deep convolutional network and demonstrating performance on the ADNI-Alzheimer's Disease Neuroimaging Initiative Dataset. We present a deep convolutional neural network to recognize Alzheimer and differentiate according the current phase of the disease.
\end{abstract}

\section{INTRODUCTION}

Senile dementia commonly known as Alzheimer's Disease, is a typical cause of dementia in the elderly. Although there is no current cure, research is underway with the development of new drugs. Alzheimer's disease has an impact on people in many ways [1]. Patients struggle with memory lapses, disorderliness, they struggle when they speak, read or write. In due course, they may not even be able to remember their relatives. They may fail to do daily tasks like brushing their teeth or even combing hair. Due to this, it causes that person to become troubled or angry or to lose their way to home. This disease can also cause death in adults. There are 3 main stages of Alzheimer's disease - moderate, mild and very mild. Diagnosis of Alzheimer remains inaccurate till the patient reaches moderate Alzheimer. However, advanced recognition as well as categorizing of Alzheimer is important for proper medical care and prevention of brain tissue impairment. More recently, deep learning strategies have led to develop high level performance in a wide range of fields also taking into consideration medical imaging analysis. We present a deep convolutional neural network for the diagnosis of Alzheimer using MRI brain data analysis. The biggest advantage in MRI scans is that they generate high- resolution localizations and, the image details are prominent for diagnostic purposes. The most obvious characteristic of Alzheimer's disease is neuron loss, followed by cerebral atrophy extending from the signature regions of the Alzheimer's disease (e.g., hippocampus and amygdala) to the entire cortical region, which MRI scan can identify.

\section{BACKGROUND STUDY AND RELATED WORK}

Making Alzheimer diagnostic classification and detection models is a very difficult task. Yet there is some remarkable research add this area. In [1] they proposed a method of multiclass classification between NC, MCI, and AD patients using multi-categorical data using deep learning and used both an ANN and a 1D CNN for this purpose. They demonstrated the effectiveness of multi-categorical data in classification as well as to compare ANNs with CNNs in this type of task. In [2] paper introduces a deep learning model for the determination of Alzheimer using Multi-Class Alzheimer's Disease and segmentation using Brain MRI Data. Design a deep convolutional network and illustrate performance in the OASIS- Open Access Series of Imaging Study database. The model is inspired by the Inception V4 network. Post preprocessing, the input is transferred to the stem layer. They rearrange the last layer of SoftMax for the diagnosis of Alzheimer's disease. The SoftMax layer consists of 4 distinct classification categories: moderate, non- demented, mild and very mild Alzheimer. The network utilizes an MRI scan as input and removes layer intelligence element representation from the first to the final exit layer. [3] This study consists of two main tests. They therefore validated an existing and highly effective method of acquiring Support Vector Machine (SVMs) as an initial trial. As those results were unsuccessful, a comprehensive learning process (Convolutional Neural Network) was proposed as a second test. [4] This study shows a group of deep-belief networks is made when the ultimate prediction is decided by the electoral system. 2 basic frameworks in deep learning and 4 different voting schemes are used and compared, due to which the development of strong structures within which discriminative elements are calculated in an unregulated manner. The resulting method was tested on a large dataset from the ADNI- Alzheimer's disease Neuroimaging Initiative. 


\section{PROPOSED APPROACH}

In this project we are using the dataset from ADNI -Alzheimer's Disease Neuroimaging Initiative database. The data obtained for this project has a directory structure with four different classes, that is, Moderate Demented, Very Mildly Demented, Very Mildly Demented, Non-Demented. On the input data preprocessing and data augmentation process is applied. In this process, we first crop the right and left part of the hippocampus present in the brain MRI images. The training dataset includes 8192 sample MRI.

images, whereas the validation dataset includes 1023 samples. The test dataset includes 1279 sample MRI images. To generalize the very specific MRI images, we apply augmentation operations in the form of rescaling, rotation, width shift, height shift, shearing, brightness changes, zooming and horizontal flipping.
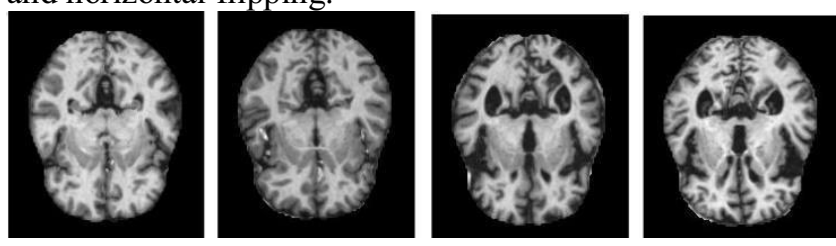

Fig 3.1: MRI Images (ADNI dataset)

For the training purpose using CNN algorithm a model is built in which every layer such as convolution layer, Relu layer, pooling layer from the model performs feature learning on the augmented data to obtain a feature map. It extracts the features using the feature detector and puts them in the feature map and preserves spatial relationship between pixels. The pooled feature map matrix is flattened into vectors and it is fed to the fully connected neural network.

Convolution Neural Network

Neural Networks are wont to classify various functions like image, sound, words. differing types of Neural Networks are used for a range of purposes, for instance to predict the sequence of words we use Recurrent Neural Networks accurately as LSTM, similarly for image classification using the Convolution Neural Network. In the next section, we are going to build a basic CNN blockchain. A standard Neural Network consists of 3 layers Input layer: The layer where we provide inserts in our model. the quantity of neurons during this layer equals the entire number of elements in our data (the number of pixels within the case of an image)

Hidden Layer: Inputs from the first layer is inserted into this layer. There may be many hidden layers counting on our data size and model. Hidden layers can have different numbers of neurons usually larger than the number of features. The output of every layer includes the matrix duplication of the previous layer by the readable weights of that layer then by inserting the readable bias followed by the activation function that produces the network out of line.

Output Layer: The output within the hidden layer was then incorporated into an entry function like a sigmoid or SoftMax that converts the output of every phase into a possible point for every phase. Data is then entered into the model and extracted from each layer is found this step is termed feed forward, and so calculates the error using error function, other common functions of cross entropy error, square loss error etc. After that, we return and detached the model with the output of the output. This step is termed back propagation which is employed to cut back losses.

CNN's biggest advantage compared to its predecessors is that it automatically acquires key features without any human guidance.

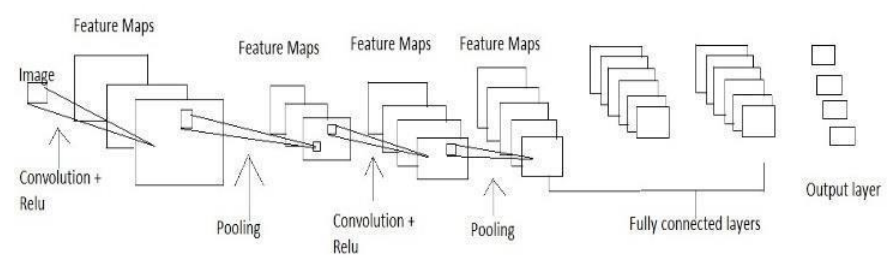

Fig 3.2: Convolutional Neural Network

Based on the extracted features it is used to classify Alzheimer Disease and its types which are: 1. No dementia 2. Very mild AD. 3. Mild AD. 4. Moderate AD

In this project, the algorithm has 3 steps. the initial step is preprocessing and then augmentation of data, the next step is extracting features from input images, and then the dementia classes are classified. The classification of dementia stages is done using a CNN based approach inspired by VGG19 for our fundamental model. The VGG19 was revised and one extra Conv layer was inserted within the model which proved efficient to know the maximum features from a small dataset. Within the algorithm, 2 modified VGG19 layers were working parallel with fourteen Conv layers, 5 max-pooling, 3 batch normalizations and 3 Gaussian noise. The rationale for the model is that the parallel work to bring out the vital features. Successive lateral layers enhanced the classification accuracy.

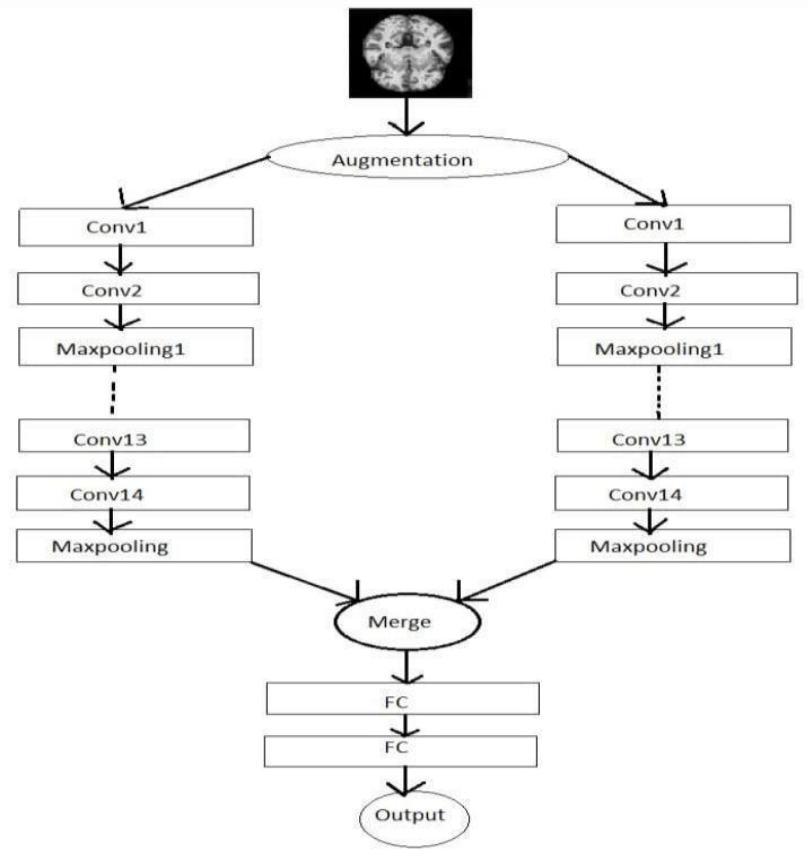

Fig 3.3: Augmentation

We implemented the dataset on VGG19 andDensenet121 and ResNet50.After evaluating the best accuracy which was obtained in modified VGG19, we deployed the model using TensorFlow, and used it in a flask-based server application for easy access for users. 


\section{IV.EXPERIMENTS AND RESULTS}

Model One: For the first model, we built a complex CNN architecture. It consists of three simultaneous layers of Conv2D and Max Pooling followed by a Flatten layer, which is again followed by two Dense Layers.

Mode1: "sequential"

\begin{tabular}{|c|c|c|}
\hline Layer (type) & Output Shape & Param * \\
\hline conv2d (Conv2D) & (None, 222, 222, 32) & 896 \\
\hline max_pooling2d (MaxPooling20) & (None, 111, 111, 32) & $\theta$ \\
\hline conv2d_1 (Conv20) & (None, 109, 109, 64) & 18496 \\
\hline $\max$ pooling $2 d \_1$ (Maxpoolingz & (None, 54, 54, 64) & $\theta$ \\
\hline Conv2d_2(Conv2D) & (None, 52, 52, 128) & 73856 \\
\hline $\max$ pooling2d_2 (Maxpooling2 & (None, 26, 26, 128) & $\theta$ \\
\hline conv2d_3 (Conv20) & (None, 24, 24, 128) & 147584 \\
\hline $\max$ pooling $2 d_{-} 3$ (Maxp $\infty 1$ ing 2 & (None, 12, 12, 128) & $\theta$ \\
\hline flatten (Flatten) & (None, 18432) & $\theta$ \\
\hline dense (Dense) & (None, 128) & 2359424 \\
\hline dense_1 (Dense) & (None, 4) & $\$ 16$ \\
\hline
\end{tabular}

Total parans: 2,600,772

Trainable parans: 2,600,772

Fig 4.1: CNN Architecture

The model was trained for 40 epochs at the end of which it had a loss of 0.0934 , and a categorical accuracy of 0.9643 for the training dataset. The graph for it as shown below:
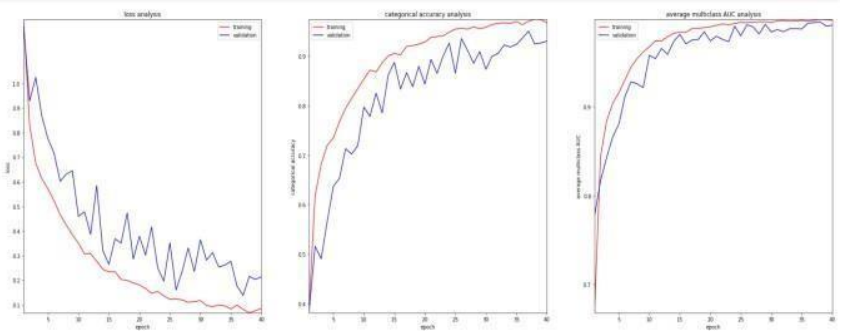

Fig 4.2: Loss and Accuracy analysis

Model Two: Next we used the Transfer Learning model. For this model, we used ResNet50 followed by several layers to modify. It had the below architecture:

\begin{tabular}{|c|c|c|}
\hline resnet5o(Functiona1) & (None, $7,7,2848$ ) & $2358771:$ \\
\hline dropout (Dropout) & (Vione, 7, 7, 2845) & e \\
\hline flatten_2 (Flatten) & (None, 16e352) & $\theta$ \\
\hline batch_nornalization (Eatchwo & (tiane, 1e9352) & 401488 \\
\hline dense_ 4 (Dense) & (twone, 64) & 6422592 \\
\hline batch_nornalization_1 (Eatch & (tione, 64) & 256 \\
\hline activation (Activation) & (Tione, 64) & $\bar{e}$ \\
\hline dropout_1 (Dropout) & (tione, 64) & 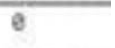 \\
\hline dense_5 (Dense) & (1wone, 64) & 4160 \\
\hline batch_normalization_2 (Eatch & (None, 64) & 256 \\
\hline activation_1 (Activation) & (1wone, 64) & $\bar{e}$ \\
\hline dropout_2 (oropout) & (Nione, 54) & $\bar{\theta}$ \\
\hline dense_f (Dense) & (None, 64) & 4160 \\
\hline batch_normalization_3 (Eatch & (ribne, 64) & 256 \\
\hline octivation_2 (Activation) & (wone, 64) & $\theta$ \\
\hline Gropout_3 (Oropout) & (1ione, 64) & $\bar{e}$ \\
\hline dense_7 (Dense) & (1vone, 32) & $2 e s e$ \\
\hline batch_normalization_4 (estch & (None, 32) & 128 \\
\hline activation_3 (Activation) & (1vone, 32) & e \\
\hline dropout_A (Dropout) & (nione, 32) & $\bar{e}$ \\
\hline dense_8 (Dense) & (Nione, 32) & 1856 \\
\hline batch_normalization_5 (Batch & (None, 32) & 128 \\
\hline activation_4 (Activation) & (None, 32) & e \\
\hline 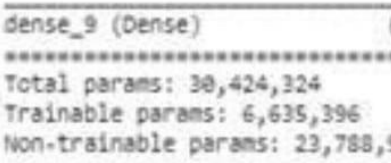 & $\begin{array}{l}\text { (None, } 4) \\
928\end{array}$ & 132 \\
\hline
\end{tabular}

Fig 4.3: Resnet50

This model was also trained for 40 epochs and showed a training loss of 0.9700 and categorical accuracy of 0.5541 which was less than what we expected so we scrapped this model for its low performance.

Model Three: This was also a Transfer Learning based model which had VGG19 as base model. It had the followed architecture: 


\begin{tabular}{|c|c|c|}
\hline Layer (type) & Output Shape & Param \# \\
\hline 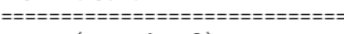 & 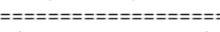 & $==========3$ \\
\hline vgg19 (Functional) & (None, 7, 7, 512) & 20024384 \\
\hline dropout_5 (Dropout) & (None, 7, 7, 512) & $\theta$ \\
\hline flatten_3 (Flatten) & (None, 25088) & $\theta$ \\
\hline batch_normalization_6 (Batch & (None, 25088) & 100352 \\
\hline dense_10 (Dense) & (None, 2048) & 51382272 \\
\hline batch_normalization_7 (Batch & (None, 2048) & 8192 \\
\hline activation_5 (Activation) & (None, 2048) & $\theta$ \\
\hline dropout_6 (Dropout) & (None, 2048) & $\theta$ \\
\hline dense_11 (Dense) & (None, 1024) & 2098176 \\
\hline batch_normalization_8 (Batch & (None, 1024) & 4096 \\
\hline activation_6 (Activation) & (None, 1024) & $\theta$ \\
\hline dropout_7 (Dropout) & (None, 1024) & $\theta$ \\
\hline $\begin{array}{l}\text { dense_12 (Dense) } \\
========================= \\
\text { Total params: } 73,621,572 \\
\text { Trainable params: } 53,540,868 \\
\text { Non-trainable params: } 20,080,\end{array}$ & $\begin{array}{l}\text { (None, } 4) \\
================ \\
, 704\end{array}$ & $\begin{array}{l}4100 \\
=========\end{array}$ \\
\hline
\end{tabular}

Fig4.4: VGG19

After training for 40 epochs, we encountered 0.2194 training loss and categorical accuracy of 0.9138 which was fairly more decent than Resnet50.

Model Four: In this, we used Densenet121 as our base model with the following architecture:

\begin{tabular}{|c|c|c|}
\hline Layer (type) & Output Shape & Param \# \\
\hline densenet 121 (Functional) & (None, $7,7,1024$ ) & 7037504 \\
\hline dropout (Dropout) & (None, $7,7,1024)$ & $\theta$ \\
\hline flatten (Flatten) & (None, 50176) & $\theta$ \\
\hline batch_normalization (BatchNo & (None, 50176) & 200704 \\
\hline dense (Dense) & (None, 64) & 3211328 \\
\hline batch_normalization_1 (Batch & (None, 64) & 256 \\
\hline activation (Activation) & (None, 64) & $\theta$ \\
\hline dropout_1 (Dropout) & (None, 64) & $\theta$ \\
\hline dense_1 (Dense) & (None, 64) & 4160 \\
\hline batch_normalization_2 (Batch & (None, 64) & 256 \\
\hline activation_1 (Activation) & (None, 64) & $\theta$ \\
\hline dropout_2 (Dropout) & (None, 64) & $\theta$ \\
\hline dense_2 (Dense) & (None, 64) & 4160 \\
\hline batch_normalization_3 (Batch & (None, 64) & 256 \\
\hline activation_2 (Activation) & (None, 64) & $\theta$ \\
\hline dropout_3 (Dropout) & (None, 64) & $\theta$ \\
\hline dense_3 (Dense) & (None, 32) & 2080 \\
\hline batch_normalization_4 (Batch & (None, 32) & 128 \\
\hline activation_3 (Activation) & (None, 32) & $\theta$ \\
\hline dropout_4 (Dropout) & (None, 32) & $\theta$ \\
\hline dense_4 (Dense) & (None, 32) & 1056 \\
\hline batch_normalization_5 (Batch & (None, 32) & 128 \\
\hline activation_4 (Activation) & (None, 32) & $\theta$ \\
\hline dense_5 (Dense) & (None, 4) & 132 \\
\hline
\end{tabular}

Fig 4.5: DenseNet

After training the model for 40 epochs, we recovered a loss of 0.7075 and accuracy of 0.8411 which is better than Resnet50 but still poorer than VGG19.

\section{v. OUTPUT}

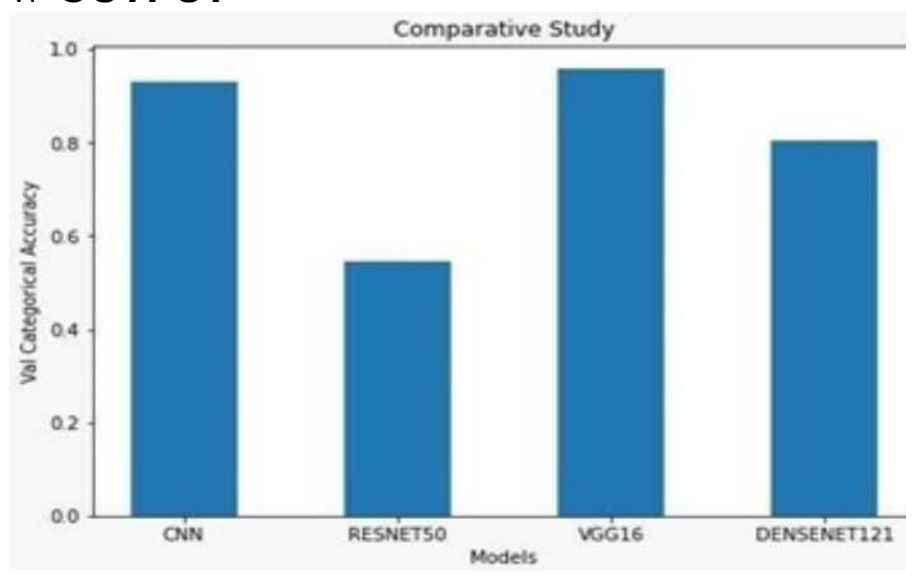

Fig 5.1: Validation Categorical Accuracy Analysis

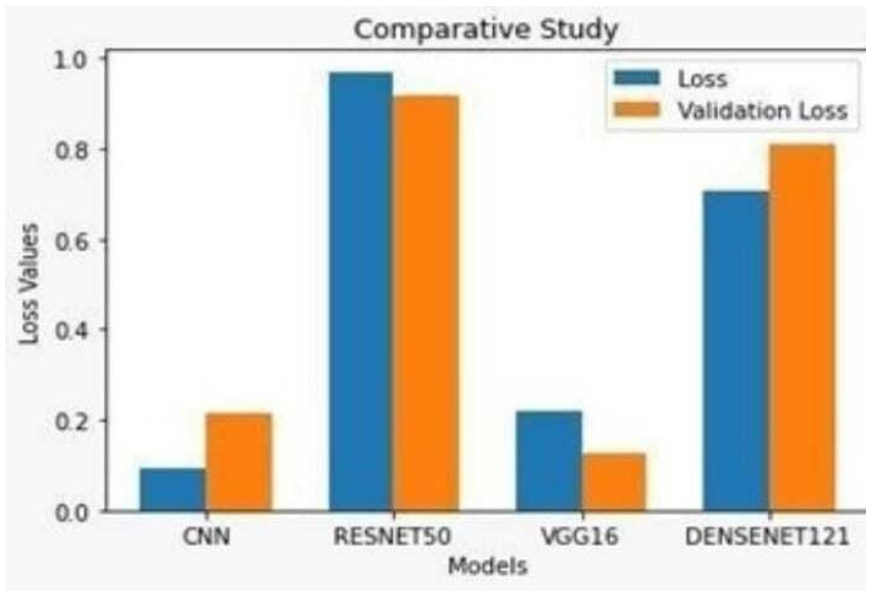

Fig 5.2: Loss Analysis

From the above graph it shows that VGG19 has the highest accuracy. Now we deploy the best model using TensorFlow so that we can use it in our website. We have created a User Application for patients, we have used Python's web framework called Flask which helps us in creating an end-toend application for patients. We have deployed TensorFlow model here to predict the outcome for the MRI images with Alzheimer's.

\section{VI.CONCLUSION}

Alzheimer is a common neurodegenerative disease among older people. Therefore, its early detection is necessary for proper treatment and to prevent mis happenings. This project helps to automate Alzheimer detection using deep learning. The main goal of this project is to come up with an efficient way for people to take the necessary and suitable precautions from having Alzheimer's disease. Using this project, we can make its diagnosis accessible to everyone if brain MRI scan is available. Deep Learning is a rapidly evolving field, and its application can be especially be used in the healthcare field to maximize the benefit of the people. 


\section{REFERENCES}

1. Cohen, David \& Carpenter, Kristy \& Jarrell, Juliet \& Huang, Xudong. (2019). Deep learning-based classification of multicategorical presenile dementia data. Current Neurobiology. 10. 141-147.

2. Yanqing, Jyoti \& Zhang, Islam. (2017). a unique Deep Learning Based Multi-class Classification Method for Alzheimer's Disease Detection Using Brain MRI Data. 213-222.10.1007/978-3-319-70772-3_20.
10.1109/M2VIP.2017.8211486.

12. Naimul Mefraz Khan ,Marcia Hon. Towards Alzheimer's Disease Classification through Transfer Learning. arXiv:1711.11117v1 [cs.CV] 29 Nov 2017.

13. Anjal D,Vindhya G B, Medha Mansi, , Muskan Kedia Mahera Alam,. Prediction of Alzheimer's Disease using Machine Learning Technique. IRJET-International Research Journal of Engineering and Technology Volume: 07 e-ISSN: 2395-0056 Issue: 05 | May 2020 .

3. N. D. Kodikara, R. N. Rajapakse and K. A. N. N. P. 14. G Stalin Babu, S N Tirumala Rao, R Rajeswara Rao. Exploring Gunawardena "Applying CNN for pre-detection of Alzheimer's of Classification Methods for Early Detection of Alzheimer's disease from structural MRI data" (2017) 24th International Disease. International Journal of Engineering and Advanced Conference on Mechatronics and Machine Vision in Practice Technology (IJEAT).8958, Volume-8 Issue-6, August 2019.

(M2VIP), 2017, pp. 1-7, doi: 10.1109/M2VIP.2017.8211486.

4. Ramírez J, Górriz JM , Munilla J, G, Ramírez J Ortiz A. 15. Chandni Naidu, Dhanush Kumar, N Maheshwari, M Sivagami, Ensembles of Deep Learning Architectures for the first Gang

Diagnosis of the presenile dementia. Int J Neural Syst. 2016 Li. Prediction of Alzheimer's Disease using Oasis Dataset. IRJETNov;26(7):1650025. doi: 10.1142/S0129065716500258. Epub International Journal of Recent Technology and Engineering Issue2016 Apr 4. PMID: 27478060.

6S3 April, 2019.

5.Zeng An, Jia Longfei, Frizzell Tory, Pan Dan Huang Yin, Song Xiaowei, Early Detection of Alzheimer's Disease Using 16. Suhad Al-Shoukry1,2, And Taha H. Rassem1 (Senior, IEEE), resonance Imaging: a unique Approach Combining Nasrin M. Makbol3 1 Faculty of Computing, University Malaysia Convolutional Neural Networks and Ensemble Learning, Pahang, College of Computing and Applied Sciences, 26300 Frontiers in Neuroscience (2020), Kuantan, Pahang, MALAYSIA Alzheimer's Diseases Detection DOI $=10.3389 /$ fnins. 2020.00259 by Using Deep Learning Algorithms: April 2020.

6.I. X. Jiang, T. Nguyen, L. Chang, R. S. Turner and 17. Mr. Amir Ebrahimighahnavieh, SuhuaiLuo Raymond Chiong Almubarak, "Early Detection of Alzheimer's Disease Using The University of Newcastle, University Drive, Callaghan 2308, Patient Neuropsychological and Cognitive Data and Machine Australia. Deep learning to detect presenile dementia from Learning Techniques," 2019 IEEE International Conference on neuroimaging: a scientific literature review. 2019.

Big Data (Big Data),Los Angeles, CA, USA, 2019.

7.Jyoti \& Zhang, Islam Yanqing. Brain MRI analysis for 18. Tong L, Venugopalan J, Wang MD, Hassanzadeh HR. Alzheimer's disease diagnosis using an ensemble system of Multimodal deep learning models for early detection deep convolutional neural network. Brain Informatics. (2018). of Alzheimers stage. doi 10.1038/s41598-020-74399-w Sci Rep. 5-10.1186/s40708-018-0080-3. 2021Feb5;11(1):3254.

8. L., Gong, X., K., Mao, Chen, M., Li, J., Nandi, Yue, \& Li, 19. Lucia Billeci, Lorenzo Bachi, Alessandro Tonacci and Asia M. (2018). Auto-Detection of Alzheimer's Using Deep Convolutional Neural Networks. 2018 14th International Conference on Natural Computation, Fuzzy Systems and Badolato. Machine Learning for the Classification of Alzheimer's Disease and Its Prodromal Stage Using Brain Diffusion Tensor Imaging Data: a scientific Review September 2020.

Knowledge Discovery (ICNC-FSKD).

20. Aunsia Khan and Muhammad Usman Dept. of Computing, 9. Qin Z, Lan T, Ding Y Zhang C, Luo C, , Xiao Z. Brain MR Shaheed Zulfikar Ali Bhutto Institute of Science and Technology Image Classification for Alzheimer's Diagnosis supported Multifeature Disease using Machine Learning Techniques A Review Paper. Fusion.Comput Math Methods Med. (2017); 2017:1952373. KDIR 2015 - 7th International Conference on Knowledge doi:10.1155/2017/1952373. Epub 2017 May 22 Discovery and data Retrieval.

10. Kim Oh, Chung K., YC., K.W. et al. Classification and 21. Kwangsik Nho, Taeho Jo, and Andrew J. Saykin. Deep Visualization of Alzheimer's Disease using Volumetric Learning in Alzheimer's Disease: Diagnostic Classification and Convolutional Neural Network and Transfer Learning.

$\begin{array}{lllll}\text { Sci } & \text { Rep } & \text { 9, } & \end{array}$

11. R N Rajapakse, N D Kodikara and K A N N P 22. Alzheimer disease facts and figures. Available online 20 Gunawardena. Applying Convolutional Neural Networks for March 2018.

Pre-detection of Alzheimer's Disease from Structural MRI

data. Conference Paper November 2017 DOI: 5 